\title{
On Chinese Translation of Long English Sentences in EST From the Perspective of English Thinking Modes
}

\author{
Zhenghua Tan \\ Jianghan University, Wuhan, Hubei Province, China \\ Xixiang $\mathrm{Ke}$ \\ Wuhan Textile University, Wuhan, Hubei Province, China
}

\begin{abstract}
Expression follows man's thinking, nature of translation is the transfer of different thinking modes, and thinking is the foundation and precondition of translation. Studying the thinking characteristic of source languages and analyzing the differences between English and Chinese help to find the solution to the problems in translation. The paper probes into the features of English language in thinking and explores the syntactic structures in EST and focuses on strategies in the process of translating long sentences in EST.
\end{abstract}

Index Terms - thinking feature, long sentence, translation strategy, EST (English for science and technology)

\section{INTRODUCTION}

The foundation of translation activities is the commonality of human thinking modes, and the essence of translation is the transfer of different thinking forms. Regarding the difference between Chinese and Western thinking styles, Fu Lei pointed out that there is a big gap between Chinese thinking and Western thinking. Westerners lay stress on abstract and analysis (Sun Zhili, 2003, p 77). The western thinking mode focuses on formal logic and is good at deduction; after the modern experimental science comes into being, it has also paid attention to induction. The combination of deduction and induction has produced countless theoretical systems in the West and greatly promoted the development of Western science. That mode, which has a strong objectivity , being good at "commentary" discussion and western scientific cognitive thinking, emphasizes exploring nature, seeking knowledge, and rationality and exploration outward (Lian Shuneng, 2002,p2). Thinking mode is the synthesis and unification of the stereotyped thinking form, thinking method and thinking procedure of the subject in the thinking process (Rong Kaiming, 1989, p30). The units of thinking are concepts, judgments and inferences, while the units of language are words, sentences, paragraphs and texts. There is a certain correspondence between thinking and language. The way of thinking determines the expression of language (Bao Huinan, 2003, p35). In the translation process, the study of the characteristics of the thinking mode of the target language is an important measure to reduce and eliminate barriers in cross-cultural communication (Chen Hongwei, 2008, p25). Long sentences in scientific and technical English literature, scientific and technological standards, patent specifications, and principle explanations are especially occur. Text features present in polysemy, long sentences, passive sentences, part-of-speech conversions, non-predicate verbs, and professionalism. They are used in scientific and technological English. Long sentences are used to describe the complex and changeable objective world, and these characteristics are all determined by the content of scientific and technological literature. In the translation of technical English, how to deal with the translation of long sentences has become the most concerned issue.

\section{THE EXPRESSION OF THE THINKING MODES OF EST IN SENTENCES}

\section{A. Prominence and Brief in a Main Structure}

English sentences draw mainly support from the grammatical means of the language to connect sentences. The main structure is short and prominent (that is, "hypotaxis" is emphasized), and the expression is straight to the point. with the help of English unique vocabulary, prepositions, conjunctions, space construction is carried out, and each part is organically combined (Chen Anding, 2006, p5). Similarly, in EST long sentences, although the sentence structure is complex, the main part is easy to identify.

Example 1

The amount of $\mathrm{N}$ released annually into the soil, mainly as dead tissue protein and as excretorymucus, urea, uric acid and ammonia, is estimated as being between 18 and $92 \mathrm{kgha}-1$, depending on the size and activity of the earthworm population (Guo Huili, 2008,p13).

The subject of the sentence "the amount of $\mathrm{N}$ " is more obvious. There are two predicate verb forms in the sentence: "released", "is estimated as". Further analysis shows that "released annually into the soil" is a past participle phrase, 
which modifies "noun" as an attribute. Therefore, the main structure of the sentence is "the amount of $\mathrm{N}$ is estimated as being between 18 and 92" Kgha-1. "The main part can be translated as:

dan de zong liang gu ji mei gong qing ke da 18dao 92gong jin。（氮的总量估计每公顷可达 18 到 92 公斤。）

Translation:

mei nian zhu yao yi shi ti dan bai zhi 、pai xie de zhan ye 、 niao su 、niao suan 、 he an de deng xing shi , you qiu yin shi fang dao tu rang de dan de zong liang , (zai ) ji yu qiu yin qun ti de da xiao bu tong he huo dong de cha yi (ji chu shang）, gu ji mei gong qing ke da 18dao 92gong jin。（每年主要以尸体蛋白质、排泄的粘液、尿素、尿酸、 和氨的等形式, 由蛢蚓释放到土壤的氮的总量, （在）基于虾蚓群体的大小不同和活动的差异（基础上）, 估 计每公顷可达 18 到 92 公斤。)

Example 2

Gas generating components, capable of quickly producing a large quantity of solids-free nontoxic gases useful as propellants in Halon replacement fire suppression systems consist of a double-based castable propellant formulated from a major amount of ammonium nitrate, a minor amount of nitrocellulose, one or more high-energy nitrate ester plasticizer, a thermal scavenger, and a combustion rate modification (Guo Huili, 2008, p13)

This is a sentence with about 60 English words. It seems long in expression, but in fact it is a simple sentence with only one subject and one predicate."capable of quickly producing a large quantity of solids-free nontoxic gases"and" useful as propellants in Halon replacement fire suppression systems"are adjective phrases as attributives(Tan\&Ke,2021,p232), therefore, they can't be the subject. The subject is "Gas generating components". The verbs that can be used as predicates in long sentences are "consist of" phrase and the phrase "formulated from"(Tan,2021,p232).But a further analysis shows us"formulated from a major amount of ammonium nitrate , a minor amount of nitrocellulose, one or more high-energy nitrate ester plasticizer, a thermal scavenger, and a combustion rate modification.(Guo Huili,2008, p13)'It's a past participle phrase. It's an attribute in a sentence, so the verb is "consist of" . Based on that, the main structure of the sentence is"Gas generating components consist of..."(Tan\&Ke,2021,p232).

According to the English sentence structure, the main part can be translated into Chinese, "sheng cheng qi ti cheng fen shi you ...zu cheng。(生成气体成分是由...组成)”

Translation:

zuo wei ha long mie huo qi ti dai xi tong tui jin ji de sheng cheng qi ti cheng fen , （ying shi ） you zhu yu kuai su chan sheng da liang wu gu ti can zha he wu du de qi ti , you cong ran shao lü xiu zheng 、 jian re zhuang zhi 、da liang xiao suan an 、wei liang de xiao hua xian wei 、yi zhong huo shu zhong gao neng xiao suan zhi ke su ji deng xiang guan zhu yin su she xiang er lai de ke jiao zhu shuang ji tui jin ji zu cheng (Tan\&Ke,2021,p232)。(作为哈龙灭火器替代系统 推进剂的生成气体成份, (应是) 有助于快速产生大量无固体残渣和无毒的气体, 由从燃烧率修正、减热装置、 大量硝酸铵、微量的硝化纤维、一种或数种高能硝酸脂可塑剂等相关诸因素设想而来的可浇铸双基推进剂组成。)

For this kind of English sentence expression structure, that is, the trunk is short and prominent, with many branches attached to the trunk, it is compared to "a towering tree", "a bunch of grapes", "a bunch of pearls" and so on.

\section{B. Uniqueness of Finite Verb in Long Sentences of EST}

The sentences in EST usually take the subject predicate structure as the main part and the predicate verb as the center. The finite verb is consistent with the subject in person and number. No matter how long a sentence is in EST, there is only one finite verb which can be used as a predicate (including a parallel predicate). If other verbs appear, they will appear in non-finite. The main idea of the sentence is expressed by the verb predicate. Therefore, to find out the finite verbs in a sentence is to grasp the main part of the sentence, and the whole sentence structure will be illustrated (Chen Ding'an, 2006, p41). The other parts are completed by non finite verb, participle, gerund, preposition, attributive, adverbial and object clauses.

Example3

We hypothesized that variation in the genes coding for phytoene synthase(Psy), a critical enzyme in carotenoid biosynthesis, may partially explain the phenotypic variation in endosperm color observed among durum cultivars(Guo Huili, 2008,p14)

First, analyze the main sentence ,it could be read: We hypothesized..., the finite verb"hypothesized"is used as verb in the sentence, and all the development expression based on "hypothesized" and extended, and that followed by object clause. The main structure of the object clause is: variation may partially explain the phenotypic variation; Prepositional phrase "in the genes" is used as adverbial; The present participle phrase "coding for phytoene synthase (Psy)" is used as attributive to modify"genes"; "a critical enzyme in carotenoid biosynthesis" is used as "Psy" appositiv; the past participate phrase "observed among durum cultivars" is used as attribute to modify“endosperm color".

Translation:

wo men ceng jia ding , bian ma ba qing fan qie hong su he cheng mei (Psy), ji yi ge zai lei hu luo bo su sheng wu he cheng guo cheng zhong de guan jian mei , zai ji yin zhong de bian yi ke yi bu fen di jie shi wei zai ying zhi xiao mai pin zhong pei yu zhong guan ce dao de ci sheng pei ru yan se de biao xing bian hua 。（我们曾假定, 编码八氢番茄 
红素合成酶 (Psy), 即一个在类胡萝卜素生物合成过程中的关键酶, 在基因中的变异可以部分地解释为在硬质 小麦品种培育中观测到的次生胚乳颜色的表型变化。）

Example 4

A widely held theory is that the cumulative acid deposition from rainfall will alter soil's chemistry, releasing such toxic metals as aluminum, which had earlier been innocuously bound in the soil (Guo Huili, 2008,p14).

Obviously, the main part of the sentence is "a wide held theory is", "is" as the main predicate; "that" followed by a subordinate clause. The main structure of the clause is "the cumulative acid disposition will alter soil's chemistry"; the present participle phrase"releasing such toxic metals as aluminum"is used as an adverbial; the attributive clause "which had earlier been innocuously bound in the soil" modifies such toxic metals.

Translation:

pu bian de guan dian ren wei , cong jiang yu zhong lei ji qi lai de suan chen ji wu , shi fang chu xiang lü zhe yang de you hai jin shu, jiang dao zhi tu rang de hua xue xing zhi fa sheng gai bian, zhe xie jin shu yuan xian shi yi wu du de xing shi xi fu zai tu rang zhong de 。(普遍的观点认为, 从降雨中累积起来的酸沉积物, 释放出象铝这样的有害 金属, 将导致土壤的化学性质发生改变, 这些金属原先是以无毒的形式吸附在土壤中的。)

It is evidently that the above two examples are centered on the predicate verb, with the help of connecting means such as participles, prepositions, conjunctions, relational pronouns and so on, the other grammatical elements of the sentence are put up layer by layer, showing a "branched" structure extending from the center to the outside. To understand and grasp the predicate verb of a long sentence is to grasp the soul of the whole sentence.

\section{Focus Preposed in the Long Sentences of EST}

In compound sentences, main sentences are the main parts, which are usually placed at the beginning of the sentences (Chen Ding'an, 2006, p11). That is to say, English sentences usually use the position of front center. This "from one to many" western way of thinking tends to focus on the beginning of a sentence (Bao Huinan, 2003, p39). In long sentences of EST, especially in compound sentences, the main part of the main sentence is placed at the beginning of the sentence. In terms of expression, it first gives the conclusion, and then elaborates it layer by layer, reflecting the analytical thinking mode of western language.

Example 5

This remains to be established in studies of these chemically modified cotton celluloses in which the amine content is varied over a wide range for each type of composition(Guo Huili, 2008,p13).

It can be seen that the underlined part is the focus of the sentence.

Translation of the main sentence: "zhe jiang you dai zai zhu hua xue gai xing de mian xian wei su yan jiu zhong yu yi que zheng. (这将有待在诸化学改性的棉纤维素研究中予以确证。) ”It is the conclusion part of expression.

Full sentence translation: zhe jiang you dai zai yan jiu ge zhong hua xue gai xing de mian xian wei su (mei yi zhong de an han liang dou you hen da bian hua ) shi yu yi que zheng 。(这将有待在研究各种化学改性的棉纤维素 (每一 种的胺含量都有很大变化) 时予以确证。)

Example 6

Thus it was possible for us to see how the well-known basic laws controlling all kinds of fluid motion, from ocean currents to water in pipes, had to be adapted to satisfy the ways of the atmosphere (Guo Huili, 2008,p14)

Similarly, the underlined part is the focus of the sentence and the conclusion part of the expression.

Full sentence translation: yin ci , wo men bian ke neng le jie na xie zhi pei ge zhong liu ti yun dong (cong da yang de yang liu dao guan zhong de shui liu ) de zhe ming ding lü bu de bu zuo xie diao jie yi quan shi da qi huo dong de ge zhong fang shi。(因此, 我们便可能了解那些支配各种流体运动（从大洋的洋流到管中的水流）的著名定律不 得不作些调节以诠释大气活动的各种方式。)

Example 7

Victory Garden, a collection of 75 essays ranging from general topics such as fertilizers and crop yield to detailed information on all the standard garden vegetables and 60 species of houseplants, is now part of Compuserve's thousands of informational categories, according to Ted Batutis, a computer consultant with a doctorate in vegetable crops (Guo Huili, 2008, p13).

First, analyze the sentence, which may be the part of the subject, one is "Victory Garden", the other is "a collection of 75 essays..." It can be seen that the latter is a development sentence explained to the former. So "Victory Garden" can be seen as the subject. If we take a close look at the predicate part and analyse grammatically, the whole structure of the main sentences is very obvious. The verb is the predicate form, that is "is part of..." It consists of the complement part. So, the underlined part (victory garden, now part of CompuServe's thousands of information categories.) that is the focus of the sentence. In terms of expression, it is also the conclusion part.

Full sentence translation: ju shu cai zuo wu xue bo shi 、 ji suan ji gu wen Ted Batutisshuo , shou lu 75pian lun wen , nei rong han gai cong fei liao he zuo wu chan liang deng pu bian xing wen ti , zai dao you guan ge zhong biao zhun de yuan pu shu cai he 60zhong shi nei zuo wu de xiang xi zi liao de 《sheng li yuan pu 》, xian zai shi Compuserveji qian zhong xin xi fen lei zhong de yi bu fen (Tan \& Ke, 2021, p232).(据蔬菜作物学博士、计算机顾问 
Ted Batutis 说, 收录 75 篇论文, 内容涵盖从肥料和作物产量等普遍性问题, 再到有关各种标准的园圃蔬菜和 60 种室内作物的详细资料的《胜利园戋》，现在是 Compuserve 几千种信息分类中的一部分。).

\section{TRanslation Principles Based on Thinking Modes of EST}

There are three thinking characteristics reflected in EST sentences: first, the main structure is short and prominent; second, the uniqueness of predicate (including coordinate predicate) and finite verb in long sentences; third, the preposition of main ideas (i.e. main sentence) in long compound sentences. Relevant suggested methods can be used in reading comprehension and translating long sentences.

\section{A. Identify Main Part and Branches.}

Based on the characteristics that long sentences are constructed on the basis of the main part with the help of Special English vocabulary, and each part is organically combined. If the main part is found out, the branches are easily done.

Example 8

It follows that the thickness of pavement material required to lower the stress from that applied by the wheel to the upper surface of the pavement, to that allowable at the under surface, depends upon the spreading ability of the pavement materials (Guo Huili, 2008, p13).

First, we analyze the main part of the sentence. We can see that the main sentence is the object clause guided by it follows + that-clause, and the finite verb used as the predicate is "follow" In the object clause"required to lower the stress" is a participle phrase to modify the "thickness of payment material"; another participle phrase"applied by...the payment" modifies the pronoun "that"; an adjective phrase "allowable at the under surface" modifies the second pronoun "that".

Translation :

pu dian cai liao de hou du yao qiu ba che lun shi yu lu mian de ya li jiang di dao di ji neng gou cheng shou de cheng du ; er zhe ge hou du ze qu jue yu gai cai liao de pu san neng li 。(铺垫材料的厚度要求把车轮施于路面的压力降 低到底基能够承受的程度; 而这个厚度则取决于该材料的铺散能力)

\section{B. Find out the Predicate Verb and Grasp the Focus of the Sentence}

In long sentences of EST, when expressing the complicated meaning, the western people tend to get to the point and demonstrate one by one. Therefore, the focus of the sentence is placed at the front, which is often the main idea of the sentence.

Example 9

It may come as a shock to mathematicians to learn that the Schrodinger equation for the hydrogen atom is not a literally correct description of this atom, but only an approximation to a somewhat more correct equation taking account of spin, magnetic dipole, and relativistic effects; and that this corrected equation is itself only an imperfect approximation to an infinite set of quantum field-theoretical equations (Guo Huili, 2008,p15).

Sentence analysis: throughout the whole sentence, remove the non-finite verb "to learn", participle "corrected", "taking account of", and the finite verb is "come". The two "is" are the predicate verbs in the clause guided by that. So the predicate is "come". According to the above discussion, the part of the sentence where the predicate verb is located is also the focus of the sentence, namely, "It may come as a shock to mathematicians".

Translation of the main part:

dui shu xue jia er yan , le jie dao zhe zhong qing xing ke neng hui ling qi jing e bu yi (对数学家而言, 了解到这 种情形可能会令其惊愕不已）。

Analysis of the subordinate parts , "to learn +that clause1"; +that clause2 are used as subject .

that clause1: the Schrodinger equation for the hydrogen atom is not a literally correct description of this atom, but only an approximation to a somewhat more correct equation taking account of spin ,magnetic dipole, and relativistic effects (Guo Huili, 2008,p15).

Translation of this part: xue ding e de qing yuan zi fang cheng shi bing fei shi dui gai yuan zi zuo chu de yi zhong zi mian shang zheng que de miao shu , er jin jin shi ba xuan zhuan 、ci xing ou ji zi 、yi ji xiang dui lun xiao ying kao lü zai nei 、zai mou zhong cheng du shang de geng wei zheng que de de fang cheng shi de yi ge jin si zhi （薛定谔的氢 原子方程式并非是对该原子做出的一种字面上正确的描述, 而仅仅是把旋转、磁性偶极子、以及相对论效应考 虑在内、在某种程度上的更为正确的的方程式的一个近似值.Guo Huili, 2008,p15）。

that clause 2: this corrected equation is itself only an imperfect approximation to an infinite set of quantum field-theoretical equations.

Translation of this part: zhe ge de yi jiu zheng de fang cheng shi jiu qi ben shen er yan ye zhi shi yi ge qu jin yu wu qiong wu jin de liang zi chang lun fang cheng shi zhong de yi ge bu wan mei jin si zhi (这个得以纠正的方程式就其 本身而言也只是一个趋近于无穷无尽的量子场论方程式中的一个不完美近似值 Guo Huili, 2008,p15）。

Full sentence translation:

dui shu xue jia er yan , le jie dao zhe zhong qing xing ke neng hui ling qi jing e bu yi , ji xue ding e de qing yuan zi 
fang cheng shi bing fei shi dui gai yuan zi zuo chu de yi zhong zi mian shang zheng que de miao shu , er jin jin shi ba xuan zhuan 、ci xing ou ji zi 、yi ji xiang dui lun xiao ying kao lü zai nei 、zai mou zhong cheng du shang de geng wei zheng que de de fang cheng shi de yi ge jin si zhi ; bing qie zhe ge de yi jiu zheng de fang cheng shi jiu qi ben shen er yan ye zhi shi yi ge qu jin yu wu qiong wu jin de liang zi chang lun fang cheng shi zhong bu wan mei de jin si zhi (对 数学家而言, 了解到这种情形可能会令其惊愕不已, 即薛定谔的氢原子方程式并非是对该原子做出的一种字面 上正确的描述，而仅仅是把旋转、磁性偶极子、以及相对论效应考虑在内、在某种程度上的更为正确的的方程 式的一个近似值; 并且这个得以纠正的方程式就其本身而言也只是一个趋近于无穷无尽的量子场论方程式中不 完美的近似值(Guo Huili, 2008, p15)。

Surely, when translating long sentences in EST, in case that the single way may be difficult to achieve the purpose, one method plus another can be applied in the translation.

\section{CONCLUSION}

Learning the transformation of two languages, to a large extent, is to learn the transformation of two ways of thinking. Studying the different characteristics of thinking between the East and the West and their linguistic expression can help us to deal with the difficulties in scientific and technological translation. All translation theories, methods and techniques are based on the comparison between English and Chinese. Therefore, from the perspective of translation practice, we can compare the similarities and differences between English and Chinese, especially the differences, analyze the similarities and differences in thinking between English and Chinese, especially the differences so that we can consciously use these characteristics in translation and find ways to solve problems. If we explore the thinking characteristics of two scientific and technological languages in sentence patterns, we may have deeper significance in solving the problem of translation of long sentences in scientific and technological English.

\section{REFERENCES}

[1] Bao Huinan. (2003). Cultural context and language translation. Beijing: China International Translation Publishing Company.

[2] Chen anding. (2006). English Chinese comparison and translation. Beijing: China International Translation Publishing Company.

[3] Chen Hongwei. (2008). New Chinese English translation course. Shanghai: Shanghai Foreign Language Education Press.

[4] Guo Huili. (2008). Chemical English translation skills. China Science and technology translation, 3, 13-15.

[5] Lian shuneng. (2002). On Chinese and Western modes of thinking. Dalian: Foreign Languages and Foreign Language Teaching. 2,17-18.

[6] Mao Ronggui. (1999). Translation skills 111. Shanghai: Shanghai Jiaotong University Press.

[7] Rong Kaiming. (1989). On modern thinking mode. Wuhan: Huazhong University of Technology Press,

[8] Shen Xiaolong. (1997). Chinese sentence pattern culture. Shanghai: Fudan University Press.

[9] Zhenghua Tan, Xixiang Ke. (2021). On EST sentence translation based on English and Chinese structures: natural linear expansion vs. reversed linear expansion", Theory and Practice in Language Studies, 4,231-232

[10] Sun Zhili. (2003). New English Chinese translation course. Shanghai: Shanghai Foreign Language Education Press.

[11] Zhang Peiji. (2000). English Chinese translation course. Shanghai: Shanghai Foreign Language Education Press.

[12] Zheng Shengtao. (2008).Bracket translation of long sentences in EST. Beijing: China Science and technology translation, 2, $42-46$.

Zhenghua Tan was born in Hubei Province, China in 1966. He received Master's degree in linguistics from Central China University of Science \& Technology, China in 2004.

$\mathrm{He}$ is currently an associate professor in the School of Foreign Languages, Jianghan University, Wuhan, China. His research interests include translation theory and practice.

Xixiang Ke was born in Hubei Province, China in 1975. She received Master's degree in linguistics from Central China University of Science \& Technology, China in 2006.

She is currently an associate professor in the School of Foreign Languages, Wuhan Textile University, Wuhan, China. Her research interests include translation theory and practice. 